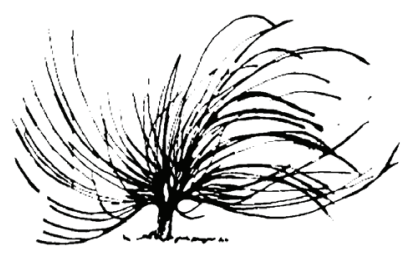

\title{
Gestión de la diversidad: Aportes para un liderazgo inclusivo
}

\author{
Karla Jiménez Porras ${ }^{1}$ \\ Centro Educativo Niño Jesús de Praga \\ Alajuela, Costa Rica \\ karla.jimenezp@gmail.com \\ Vanessa Jiménez Porras ${ }^{2}$ \\ Universidad Técnica Nacional \\ Alajuela, Costa Rica \\ vane.jp8@gmail.com
}

\begin{abstract}
Resumen
La diversidad es un reto de la realidad educativa. El presente ensayo pretende analizar las características de las escuelas inclusivas que acogen la diversidad; presta especial atención a los aspectos relacionados con la gestión de procesos educativos. Algunas características contempladas son el liderazgo compartido, el aprendizaje dialógico, el trabajo colaborativo como agente de mejoramiento escolar, la comunicación asertiva y horizontal, la reestructuración de las relaciones de poder y la promoción de valores éticos. Al mismo tiempo se contemplan ciertas prácticas que favorecen la creación de una cultura
\end{abstract}

\section{(ब)(1) (3)}

Recibido: 23 de enero de 2016-Aprobado: 25 de abril de 2016

1 Coordinadora del Departamento de Inglés y docente del Centro Educativo Niño Jesús de Praga, Alajuela, Costa Rica. Licenciada en Administración Educativa, Universidad Estatal a Distancia. Cursando la Maestría en Educación con énfasis en el Aprendizaje del Inglés, Universidad Nacional, Heredia, Costa Rica.

2 Profesora de Cursos Libres de Inglés, Universidad Técnica Nacional. Alajuela, Costa Rica. Licenciada en Docencia, Universidad de San José. Cursando la Maestría en Educación con énfasis en el Aprendizaje del Inglés, Universidad Nacional, Heredia. 
inclusiva con el fin de influenciar favorablemente el contexto y la sociedad.

Palabras clave: Inclusión, diversidad, gestión, liderazgo, colaboración, ética, comunicación asertiva.

\begin{abstract}
Diversity is a challenging reality. This essay aims to analyze characteristics present in inclusive schools, in order to address diversity, taking into consideration aspects directly related to school management. Some of these aspects are shared leadership, dialogic learning, collaborative work as an agent of school improvement, assertive communication, reformulating power relationships and the promotion of ethical values. At the same time, certain practices are considered to support the creation of an inclusive culture in order to positively influence context and society as a whole.
\end{abstract}

Keywords: inclusion, diversity, management, leadership, collaboration, ethics, assertive communication

\title{
Introducción
}

El tema de la diversidad en la población estudiantil se ha venido discutiendo desde hace muchos años, ya que representa un reto importante para todos los individuos involucrados en los procesos de aprendizaje. Gran cantidad de investigaciones y docentes coinciden en que la mayoría de las escuelas no toman en cuenta las condiciones y diferencias de su población para gestionar el proceso educativo, lo cual agudiza las desigualdades y plantea mayores retos en el aprendizaje. Según Murillo, Krichesky, Castro y Hernández (2010), "la escuela y sus docentes no están preparados para trabajar con grupos heterogéneos de estudiantes. De esta manera, aquellos alumnos que pertenecen a alguna minoría... suelen tener mayores dificultades, no sólo en términos de resultados académicos, sino también en lo referente a la participación activa en la vida escolar" (p. 170).

Dentro de este contexto, contemplar el derecho humano de recibir educación de calidad se hace cada día más complejo y, al mismo tiempo, más necesario. Se observa la necesidad de plantear respuestas 
conjuntas, por parte de todos los actores involucrados, dentro de las que se destaca el rol del liderazgo para conseguir una educación inclusiva y alcanzar la equidad.

No es posible hablar de calidad en educación pensando solamente en resultados académicos. La calidad educativa significa, principalmente, lograr la satisfacción de las expectativas y las necesidades de sus aprendientes. De esta forma, la concepción de una escuela inclusiva que se adapta a las diferencias adquiere hoy más actualidad que nunca.

Nos encontramos frente a una escuela que debe responder a las múltiples necesidades que la sociedad le demanda, estando dentro de ellas la diversidad, cada vez más visible, entre las personas. "A través de la diversidad se llega a una igualdad que no es homogénea, sino a una igualdad de derechos y oportunidades para personas diferentes" (Gómez, 2012, p. 41). Todas las personas son diversas y esto, paradójicamente, es lo que las hace iguales. La igualdad incluye el derecho de las personas a ser diferentes y a no ser asignadas a categorías ni etiquetadas de forma permanente.

La inclusión es un proceso orientado a brindar una respuesta apropiada a la diversidad de características y necesidades del estudiantado para incrementar sus niveles de logro y participación en las experiencias de aprendizaje, así como también en la cultura y en las comunidades escolares. La educación inclusiva busca "reducir toda forma de discriminación, a fin de conseguir resultados académicos equiparables con la de la mayoría de los alumnos. Desde esa perspectiva, la inclusión representa un impulso fundamental para avanzar hacia una educación de mejor calidad para todos" (Murillo, Krichesky, Castro y Hernández, 2010, 171).

Una de las exigencias más importantes en las aulas costarricenses es la atención a los sujetos aprendientes con necesidades educativas especiales. Estos grupos de estudiantes son aquellos que presentan "algún problema de aprendizaje a lo largo de su escolarización y que demandan... un abordaje educativo especial para el desarrollo de sus capacidades, lo que les permita su incorporación social" (Soto e Hinojo, 2004, p. 185). Al mismo tiempo, se busca que sean incluidos dentro del sistema educativo regular. Existen leyes y normativas que apuestan a que "el sistema educativo brinde las herramientas para que puedan alcanzar, dentro del mismo sistema, los objetivos establecidos con carácter general para todos los alumnos" (Gómez, 2012, p. 60). 
Según Jiménez (2014), “el respeto a la diversidad empieza por la tolerancia, la solidaridad y la participación en el quehacer de todos, con la fuerza y el compromiso requeridos para extraer de la diversidad cultural toda la riqueza que en ella subyace" (p. 7). La presencia de la diversidad en la población escolar cuestiona las creencias tradicionales sobre la organización y la gestión de los centros educativos, planteando "la posibilidad de un nuevo plan de acción, el cual implica nuevas consideraciones estratégicas, estructurales y personales en la gestión de las escuelas" (León, 2012, p. 136). Tomar en cuenta la diversidad es valorarla, visualizarla dentro de la planificación institucional y concientizar sobre ella a todas aquellas personas que participan de los procesos de aprendizaje en el centro educativo.

La inclusión escolar otorga un valor positivo a la diferencia, considerando que contribuye al desarrollo del ser humano. De esta forma, se constituye como "un modelo educativo que se basa en principios tales como el respeto a la diferencia y a los derechos humanos; lo que supone hablar de una cultura de la diversidad, que no es más que hablar de democracia, de convivencia y de humanización" (Gómez, 2012, p. 28).

A partir de los conceptos hasta aquí expuestos, las investigadoras consideran la inclusión escolar como la forma más adecuada de entender, vivir y practicar la educación, porque todas las personas tienen derecho a una educación de calidad, sean cuales sean sus características individuales, ya que en ellas está el desarrollo de la totalidad de estudiantes, docentes y el de la propia escuela. Tenemos la certeza que "otro tipo de educación es posible" (Gómez, 2012, p. 28).

La inclusión escolar es un ideal de la educación, que evoluciona aceleradamente. Los centros educativos deben estar preparados para atender las diferencias de todo el estudiantado, porque siempre debemos tener presentes que todos los seres somos diferentes y que en nuestras diferencias radica el aprendizaje, y el crecimiento personal y profesional.

Los centros educativos deben integrar la diversidad y convertirla en un contenido de aprendizaje más y, al mismo tiempo, deben propiciar la formación de una ciudadanía con las suficientes capacidades y habilidades que le permitan desenvolverse en la diversa y compleja sociedad que le rodea. Como consecuencia de esta doble funcionalidad, los centros educativos se ven en la necesidad de sustituir las fórmulas tradicionales de gestión escolar, por otras que busquen respuestas 
contextualizadas a las interrogantes y las demandas que día tras día la sociedad le presenta.

Sin embargo, el terreno sigue siendo confuso en cuanto a qué medidas deben tomarse a fin de avanzar en las políticas y las prácticas inclusivas. Por lo tanto, se considera de suma importancia explorar las diferentes vías para mejorar la educación hacia caminos más inclusivos. De este modo, la gestión escolar y el liderazgo juegan un papel fundamental para llegar a cumplir estos desafíos.

\section{Creación de una cultura inclusiva}

La inclusión es la clave para construir una adecuada educación en la diversidad, un proceso amplio y dinámico de construcción y reconstrucción de conocimiento que surge a partir de la interacción entre personas distintas en cuanto a valores, ideas, percepciones, intereses, capacidades, estilos cognitivos y de aprendizaje y otras características y que, según Gómez (2012, p. 33):

Favorece la construcción, consciente y autónoma, de formas personales de identidad y pensamiento, y que ofrece estrategias y procedimientos educativos variados y flexibles con la doble finalidad de dar respuesta a una realidad heterogénea y de contribuir a la mejora y el enriquecimiento de las condiciones y relaciones sociales y culturales.

Ejercer una labor transformadora en el contexto escolar no es una tarea fácil ni se logra en el corto plazo; es una acción que requiere de un proceso sistemático y critico sobre la realidad (Mencía, 2009). "Las transformaciones que se desean generar en la escuela deben partir de procesos de reflexión, de análisis individuales y colectivos" (Mencía, 2009, p. 9).

La diversidad constituye un valor y un reto continuo para la escuela inclusiva. La capacidad de liderar requiere de la organización y la creación, en forma conjunta y tras una compleja interacción entre los diferentes grupos e individuos, de una cultura inclusiva (León, 2012), entendiendo como tal: "aquella centrada en crear una comunidad segura, acogedora, colaboradora y estimulante, en la que cada uno es valorado" (León, 2012, p. 137). Pretende desarrollar valores inclusivos, 
compartidos por todo el profesorado, el estudiantado, el personal administrativo y las familias, que los transmiten a todas las nuevas generaciones de la comunidad educativa.

En una cultura inclusiva, sus miembros consideran la diversidad como oportunidad de aprendizaje, fuente de conocimientos, y vehículo de desarrollo y enriquecimiento de cada miembro de la comunidad educativa. En ella, los objetivos van más allá de alcanzar conocimiento académico, lo que presupone una nueva concepción de currículo. Se promueve el trabajo cooperativo y colaborativo entre estudiantes, entre docentes, y entre estudiantes y docentes; y la evaluación de los procesos de aprendizaje también contempla la colaboración, las actitudes y valores observados, y los objetivos no académicos (Gómez, 2012).

Las escuelas inclusivas buscan la mejora continua mediante el establecimiento de un sentido de comunidad y determinando las barreras para el aprendizaje y la participación, con el fin de eliminarlas y mostrar vías para el cambio, hacia una "cultura más inclusiva" (León, 2012, p. 138). Esta se basa en una filosofía democrática e igualitaria, incluyendo a todas las personas implicadas en la educación, en la planificación y en la toma horizontal de decisiones, de manera que puedan desarrollarse redes de apoyo para integrar a toda la comunidad, adaptar el currículo a las necesidades y mantener la flexibilidad en las estrategias y planificación curricular.

La cultura escolar inclusiva se alcanza cuando padres y madres, estudiantes, docentes y personal administrativo entablan lazos afectivos y de compromiso firme. Al mismo tiempo, las personas líderes de las escuelas inclusivas poseen habilidades y desarrollan tareas dirigidas a potenciar, en sus miembros, los conocimientos, las destrezas y las habilidades que les permitan cumplir con éxito los objetivos propuestos, mostrando una actitud de confianza en sí y en sus capacidades (León, 2012). Surge entonces la interrogante, ¿quién es líder en una escuela inclusiva?

\section{Gestión de la diversidad: Liderazgo inclusivo}

Una gestión que valore el rendimiento académico sobre el desarrollo integral de las personas promoverá acciones y políticas de competitividad que generan exclusión. Sin embargo, una escuela que valore la diversidad, la equidad y la formación humanística, priorizará tareas 
con el fin de incluir a cada participante en el éxito escolar. A este último estilo de gestión se le ha denominado "liderazgo inclusivo" (León, 2012, p. 137).

El liderazgo se entiende como una función inherente a todo grupo y, por consiguiente, a toda institución. Por eso, cada vez más autores y autoras hablan más de liderazgo y menos de líder. "Debe asumirse como una función patrimonio del grupo, no de una persona, y menos aún de un cargo en el organigrama de un centro. Constituye uno más de los valores que forman parte de la cultura de la organización" (Gómez, 2012, p. 160).

El liderazgo que hoy demandan las escuelas no está centrado en las estructuras formales, sino que se apoya en la participación y colaboración de la comunidad, donde todas las personas son líderes, o gestoras de procesos, en algún momento. Existen muchos agentes individuales o grupos que disponen de poder en una organización, según su personalidad, su carisma, sus conocimientos técnicos, su ideología, sus esfuerzos conjuntos... Esta capacidad de influencia es un poder legítimo, de carácter informal, establecido según las características particulares del sujeto o grupo, así como de su experiencia, sus conocimientos, sus destrezas de comunicación y otros. Surge en los grupos a través de relaciones espontáneas entre miembros de la organización (Gómez, 2012).

Las organizaciones educativas inclusivas requieren gestores que desarrollen acciones participativas, impulsoras y coordinadoras de la actividad pedagógica, que intervengan en la resolución de conflictos, y que desempeñen roles de "dinamizador de grupo, de mediador, de impulsor del desarrollo curricular y de la innovación en los centros" (Gómez, 2012, p. 33). A través del liderazgo distribuido (Harris, 2008, citado por Gómez, 2012) se desarrolla una nueva visión de escuela, donde se potencia la construcción de significados y el concepto de comunidad inclusiva.

Gestionar adecuadamente los procesos a lo interno del centro educativo es fundamental para atender la diversidad y lograr la inclusión. Para ello, el liderazgo inclusivo no necesariamente recae en las personas que ostentan un cargo administrativo, sino que puede ser llevado a cabo por un colectivo de individuos que trabajan juntos, que comparten iniciativas, y que construyen la cotidianidad del centro a partir de este trabajo colaborativo, de acuerdo con Groon (2003, citado por Murillo, Krichesky, Castro y Hernández, 2010). 
El liderazgo inclusivo debe estar centrado en una gestión democrática y participativa, en la cual son prioritarias: dinámicas de indagación abierta, pedagogía crítica, comunicación asertiva en múltiples direcciones, diálogo, colaboración, empatía, y trabajo en equipo dentro de un marco de respeto en el que confluirán perspectivas diferentes (incluso a veces antagónicas), sobre las que se deberá actuar sobre una base moral y ética (Murillo, Krichesky, Castro y Hernández, 2010). Para lograr lo anterior se debe comprender que el contexto es fundamental, pues este es el espacio físico donde se asumen los valores institucionales compartidos y la toma de decisiones colectivas.

En resumen, el liderazgo inclusivo está comprometido con los valores de la inclusión y con la responsabilidad expresa de que todo el estudiantado debe aprender y participar de la vida académica y social del centro. Así mismo, su estilo de gestión debe caracterizarse por incentivar a las distintas personas que están implicadas en el aprendizaje estudiantil a participar tanto en las prácticas educativas como en la gestión de procesos, lo que nos conduce al concepto de liderazgo compartido (León, 2012).

\section{Liderazgo compartido y trabajo colaborativo}

El líder inclusivo debe ser capaz de "aprehender la naturaleza y los procesos humanos básicos con el fin de conocer el potencial y los límites del cambio social" (Mencía, 2009, p. 86). Las interacciones, señala Mencía (2009) siempre son recíprocamente educativas y ningún liderazgo logra imponerse, si no responde a las necesidades y situaciones de los demás. Por ello, debe producir consecuencias significativas, lo que reconoce la importancia de la participación de la comunidad.

Para que la escuela sienta la obligación de atender las demandas de la sociedad, se hace necesario diseñar acciones que partan de "la colaboración de todos los miembros de la comunidad educativa, creando un sentimiento de comunidad que facilite el éxito de sus miembros" (Gómez, 2012, p. 147). Esto conlleva una apuesta fuerte por el liderazgo compartido, donde no existe un solo líder y donde quienes lo ejercen muestran independencia del liderazgo formal (director o directora), de tal forma que toda la comunidad educativa participe en este. Deben hacerlo desde posiciones personales y sociales que les permitan "un acercamiento al trabajo de los demás miembros de la comunidad educativa, 
con respeto ante posturas diferentes y en una actitud de diálogo permanente" (Gómez, 2012, p. 162).

Los estudios recientes sobre gestión en las escuelas apuntan hacia el liderazgo distribuido, eliminan la identificación de esta función exclusivamente con el equipo directivo del centro, y comienzan a concebir que el liderazgo puede ser compartido a través de una estructura de gestión en todos los niveles en la comunidad escolar. Se destaca el "liderazgo comprometido y distribuido como la única concepción del líder que se ajusta a la escuela como organización que aprende, como comunidad profesional de aprendizaje y como escuela inclusiva" (Gómez, 2012, p. 161).

Esta idea de liderazgo está fundamentada en cinco supuestos, según León (2012, p. 38):

- $\quad$ Liderazgo y líder no son lo mismo.

- $\quad$ El liderazgo busca el aprendizaje que conduce a un cambio constructivo.

- Toda persona tiene el potencial y el derecho a trabajar como líder.

- Liderar es un esfuerzo compartido, a partir de la democratización de la escuela.

- $\quad$ El liderazgo requiere la redistribución de poder y de autoridad.

Las prácticas asociadas al liderazgo compartido, según León (2012), incluyen la construcción de una visión de escuela compartida; fomentar la aceptación de objetivos grupales, y demostrar altas expectativas sobre la calidad y el cumplimiento de las propuestas. De esta forma, deben empezar a sustituirse las estructuras jerárquicas por la responsabilidad compartida, en una comunidad inclusiva que pasa a caracterizarse por valores y esperanzas comunes.

El trabajo colaborativo que implica el liderazgo compartido surge cuando se reúnen capacidades, conocimientos, experiencia práctica y perspectivas convergentes de quienes son miembros de la comunidad educativa, con el fin de resolver problemas cotidianos que requieren recursos y plantean exigencias mayores que los individuos aislados. Una comunidad inclusiva valora la participación de todas las personas, ya que la diversidad es fuente de conocimientos (diversidad de docentes, de estudiantes, de contextos, de experiencias y conocimientos). Por lo 
tanto, esta labor enriquecedora permite encontrar más y mejores respuestas a los desafíos encontrados, lo que a su vez genera mayor compromiso, motivación y participación de los actores sociales.

En resumen, el liderazgo compartido se define como la función de dinamización de un grupo o de una organización para generar su propio crecimiento en función de una misión o proyecto compartido, a partir de retos o necesidades comunes (Murillo, Krichesky, Castro y Hernández, 2010, p. 175). Refuerza el sentido de comunidad y confianza mutua, al observar la necesidad de cambiar las prácticas convencionales y adaptarse a las necesidades particulares que plantea la diversidad. Esto hace que el contexto cobre un mayor sentido, ya que facilita los procesos de mejora. Asimismo, un proceso que está inherentemente relacionado con el liderazgo compartido y potencia sus alcances dentro de la organización educativa es la eficacia de la comunicación.

\section{Comunicación asertiva y aprendizaje dialógico}

La comunicación se convierte en el recurso esencial para la gestión de procesos inclusivos. Es la herramienta con la que los individuos miembros de la comunidad logran relacionarse con los demás y transmitir la visión, la misión, los objetivos y las necesidades de la escuela.

Un proceso de liderazgo compartido presupone el aumento de la autonomía en los centros escolares, el cual podría aprovecharse para resolver internamente los problemas de comunicación entre los actores del proceso de aprendizaje y para el desarrollo de conductas colaborativas, basadas en la comunicación asertiva. La comunicación horizontal y en múltiples vías puede dar lugar a grandes cambios en las comunidades escolares, convirtiéndose en un factor decisivo para atender la diversidad y las necesidades que provienen de ella (Gómez, 2012).

Lograr aprendizajes basados en valores sustentados en la inclusión y la comunicación asertiva nos conduce a la sustitución de la transmisión impositiva de información por el diálogo igualitario entre todas las personas y colectivos implicados en la tarea educativa. Tan importante es la participación de sus miembros como el diálogo entre individuos para construir aprendizajes.

Por otra parte, el diseño de un currículo contextualizado e inclusivo como una construcción colectiva favorece que su elaboración sea el producto de un consenso social entre docentes, estudiantes, personal 
administrativo y sociedad civil. De esta manera, este proceso de diseño curricular participativo a través de una comunicación fluida y permanente, permite el cambio ideológico de un currículo basado en el pensamiento de las élites académicas, a un currículo culturalmente pertinente a la población y a la diversidad (Mencía, 2009, p. 48). De esta forma, se eliminan los espacios para la exclusión y cada participante tiene la oportunidad de expresarse y de desarrollar sus capacidades de acuerdo con sus necesidades.

Finalmente, según la concepción dialógica del aprendizaje, "para aprender, las personas necesitamos de situaciones de interacción" (Gómez, 2012, p. 40). Sin embargo, no solo necesitamos interacciones, sino además, el diálogo que se establezca debe estar basado en una relación de igualdad, lo que significa que cada quien tiene conocimientos por aportar. La comunicación dentro de la cultura inclusiva reconoce la inteligencia cultural en todas las personas, quienes son capaces de transformar las relaciones, el entorno y sus propios conocimientos a través del diálogo.

\section{Comunidades profesionales de aprendizaje}

La teoría de la identidad social, según Mencía (2009), sostiene que "el ser humano experimenta un proceso cognitivo en virtud del cual se categoriza y define a sí mismo en función de su pertenencia o no pertenencia a determinados grupos sociales. Este mecanismo básico modela su percepción de la realidad y la interacción social que hace posible el comportamiento de los grupos" (p. 91). La pertenencia a una comunidad estimula el aprendizaje de todos sus miembros.

De esta manera, se podría afirmar que las escuelas donde se desarrolla una cultura inclusiva constituyen comunidades profesionales de aprendizaje, las cuales estimulan el desarrollo de las capacidades pedagógicas de sus docentes, refuerzan la coherencia y contextualización del plan de estudios, incrementan el aprendizaje instrumental y dialógico, la cooperación y la solidaridad, y aumentan el sentido de responsabilidad sobre el aprendizaje de sus estudiantes (Gómez, 2012).

Definir comunidades de aprendizaje implica continuar teorizando sobre aprendizaje dialógico, el cual resulta de las interacciones que produce el diálogo igualitario, entre iguales, para llegar al consenso, con pretensiones de validez (Valls, 2000, citado por Gómez, 2012). En 
dichas interacciones participa el conjunto de la comunidad educativa, soñando la escuela que pretende lograr y organizándose a través de un proceso diseñado con forma conjunta.

Las escuelas inclusivas, concebidas como comunidades de aprendizaje con liderazgo compartido, apuestan por la inclusión social y educativa de sus estudiantes. Son una respuesta que presupone un beneficio para toda la comunidad, ya que repercute en mejores aprendizajes para la totalidad de participantes (docentes, estudiantes, padres y madres), desarrolla una mejor convivencia y una transformación personal hacia la solidaridad y la empatía. Asimismo, promueven la igualdad de oportunidades, al convertir las aulas en grupos interactivos donde todas las personas son iguales y donde se fomenta la colaboración y la tutoría (Gómez, 2012).

\section{Relaciones de poder en la cultura inclusiva}

Según Mencía (2009), la única forma genuina de promover el desarrollo personal en la escuela es crear un ambiente de cordialidad y confianza que permita al estudiantado sentir seguridad, aceptación y valoración. La labor de las escuelas inclusivas es ofrecer estas condiciones a partir de una posición comprometida, horizontal, dialogante y democrática. Giroux (1998), citado por Mencía (2009), señala que:

Los alumnos y alumnas necesitan oportunidades de ejercitar el poder, definir aspectos sobre el currículo, controlar las condiciones de aprendizaje, y que se les permita comprometerse en sistema de autocrítica y crítica social sin miedo al castigo, así como situarse más como agentes que como objetos de conocimiento y poder. (p. 3)

La labor de la escuela inclusiva ha de estar comprometida con la generación de aprendizajes significativos y situaciones de aprendizaje que le devuelvan al estudiantado "la autonomía, la responsabilidad, la conciencia sobre sí y sobre la realidad el sentido del trabajo, ante una sociedad que niega a las mayorías la capacidad de ser sujetos, la capacidad de pensar, saber y decidir sobre su vida" (Mencía, 2009, p. 13). 


\section{Inclusión e ideología}

Según Mencía (2009), "es preciso tomar conciencia de que la práctica pedagógica es una acción política-ideológica que va a contribuir a reproducir las desigualdades de la sociedad, o por el contrario, va a ayudar a construir identidades críticas capaces de iniciar procesos de transformación" (p. 9). Una de las metas de la escuela inclusiva es formar parte del grupo que transforma la realidad, a partir del contexto inmediato, de la toma de decisiones en conjunto, para aportar soluciones a problemas cotidianos. La visión de la realidad se moldea en la escuela, la cual forma en valores y en normas para la vida en sociedad.

Las relaciones entre participantes del proceso educativo se consideran laboratorio para la sociedad y, en consecuencia, pueden concretar la formación para la convivencia, la solidaridad, el respeto, la aceptación del otro ser, la tolerancia y la concertación, haciendo legítimas las diferencias mas no la individualización, promoviendo al individuo crítico y reflexivo, para poder proyectar el futuro (Mencía, 2009). Concebidas desde esta perspectiva, las escuelas inclusivas no son instituciones que reproducen la ideología dominante, sino agentes decisivos en su construcción.

El currículo diseñando dentro de las condiciones detalladas en este ensayo debe reconocer sus propias raíces en la cultura, la historia y los intereses sociales a partir del contexto inmediato. En consecuencia, no homogenizará esa cultura, historia y necesidades, ni a sus estudiantes, sino que atenderá la diversidad de los sujetos individuales y colectivos a los cuales están destinados y se someterá a continuas revisiones, en virtud del permanente cambio al que debe responder con ética y celeridad (Mencía, 2009).

\section{Estrategias para lograr una cultura escolar inclusiva por medio del liderazgo compartido}

A partir de los estudios consultados, especialmente Gómez (2012), León (2012), Mencía (2009) y Murillo, Krichesky, Castro y Hernández (2010), se resumen ciertas estrategias para lograr una cultura inclusiva por medio del liderazgo compartido de los actores sociales. 
- Velar que los programas educativos promuevan aprendizajes significativos, dentro de un modelo inclusivo para la mejora de la calidad de vida.

- $\quad$ Disponer de planes de acogida para todo el estudiantado y sus familias.

- Desarrollar un plan de accesibilidad total que garantice que sus aprendientes dispongan de las medidas de acceso al currículo y de un plan de apoyo individualizado, teniendo en cuenta sus necesidades, expectativas personales y entorno socio-familiar y cultural.

- Fomentar el desarrollo de buenas prácticas a través de grupos de apoyo y de mejora para el desarrollo de modelos inclusivos.

- Garantizar, en la asignación de profesionales, los perfiles adecuados a cada tarea.

- Proporcionar a todos los alumnos y alumnas un programa educativo que se desarrolle en un entorno plural y multicultural.

- Fomentar el trabajo en equipo, garantizando los espacios y tiempos que lo hagan posible, promoviendo la reflexión y el debate sobre conductas y actitudes éticas.

- Procurar métodos para favorecer la aceptación, por parte de sus iguales, del alumnado con necesidades educativas especiales.

- Determinar cuáles son las técnicas de clase más efectivas para trabajar con la diversidad.

- Promover métodos para facilitar la cooperación entre pares y entre docentes.

- Mejorar la eficacia del profesorado de educación general con estudiantes que presentan necesidades educativas especiales.

- Incentivar actitudes de respeto, empatía y solidaridad entre miembros de la comunidad educativa.

\section{Consideraciones finales}

El liderazgo inclusivo, como estrategia para acoger y aprovechar la diversidad existente con el fin de generar conocimiento que nutra a la totalidad de miembros de la comunidad escolar, fomenta también la creación de comunidades de aprendizaje en las que se promueve un sentido de pertenencia entre sus miembros, profesorado, alumnsdo y familias. Promueve la construcción en conjunto de una cultura diversa e inclusiva, que potencia el capital social de sus estudiantes. 
En este sentido, el liderazgo compartido se lleva a cabo por líderes capaces de asumir riegos, accesibles a diferentes miembros de la comunidad educativa, porque forman parte de ella en una relación horizontal. Son sujetos reflexivos e intencionales y comparten el liderazgo con otros individuos o grupos de la organización, sabiendo que el hecho de compartir los objetivos inclusivos les convierte en líderes que promueven eficazmente la equidad y el respeto de todas las personas.

Es de vital importancia, para que este proceso de liderazgo logre construir una escuela más inclusiva, que los actores de los procesos de aprendizaje se involucren en la toma de decisiones y en la investigación de su propio contexto, con el fin de seguir indagando acerca de cómo trabajan, qué sienten, cómo piensan y qué estrategias pueden adoptar para poder dar respuesta a los desafíos que la diversidad plantea en las aulas y en las escuelas.

Finalmente, se ha de afirmar que queda un largo camino por recorrer en aras de lograr escuelas verdaderamente inclusivas en Costa Rica. Sin embargo, desde la práctica y la investigación, es posible que poco a poco logremos transformar nuestras instituciones e, idealmente, nuestra sociedad, en comunidades más justas, equitativas e inclusivas.

\section{Referencias}

Gómez, I. (2012). Dirección escolar y atención a la diversidad: Rutas para el desarrollo de una escuela para todos [Tesis de doctorado]. Universidad de Huelva. Huelva, España.

Hernández, M. L. (2013). Liderazgo académico. Revista de la Educación Superior, 42(3), 105-131.

Jiménez, K. (2014). Análisis de la gestión educativa y su repercusión en la inclusión y educación integral de los (as) estudiantes del Centro Educativo Niño Jesús de Praga, del Circuito 02, de la Dirección Regional de Educación Alajuela, durante el curso lectivo 2012 [Tesis de licenciatura]. Universidad Estatal a Distancia. San José, Costa Rica.

León, M. J. (2012). El liderazgo para y en la escuela inclusiva. Revista Educatio Siglo XXI, 30(1), 133-160.

Mencía, P. (2009). Las relaciones de poder entre docente-alumnos y alumnas en un aula urbana de $5^{\text {to }}$ grado del distrito educativo 
08-03 Santiago, República Dominicana [Tesis de doctorado]. Universidad Estatal a Distancia. Santiago, República Dominicana. Murillo J., Krichesky, G., Castro, A. y Hernández, R. (2010). Liderazgo para la inclusión escolar y la justicia social. Aportaciones de la investigación. Revista Latinoamericana de Inclusión Educativa, 4(1), 169-186. Descargado de http://www.rinace.net/rlei/numeros/vol4-num1/art8.pdf

Soto, R. e Hinojo, F. J. (2004). La colaboración entre maestros/maestras y padres/madres para atender a la diversidad en las instituciones educativas. Revista Educación, 28(2), 185-201. 